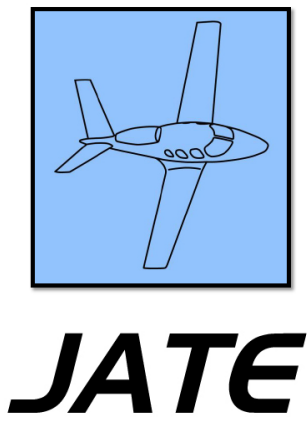

Journal of Aviation Technology and Engineering 7:1 (2017) 58-65

\title{
Advantages and Disadvantages of Using Internet-Based Survey Methods in Aviation-Related Research
}

\author{
Stephen Rice, Scott R. Winter, Shawn Doherty, and Mattie Milner \\ Embry-Riddle Aeronautical University
}

\begin{abstract}
Within the last decade, numerous online populations, such as SurveyMonkey ${ }^{\circledR}$ and Amazon's Mechanical Turk ${ }^{\circledR}$ (MTurk), have been established allowing researchers to gather data involving diverse populations. These resources offer an alternative to traditional laboratory settings hosted at universities, where many studies utilize students as the available and accessible population. While these online portals do provide new opportunities, they also contain unique advantages and disadvantages. This paper synthesizes the advantages and disadvantages of using online populations to conduct research in the aviation field. Some of the advantages are: easier access to new populations, larger sample sizes, more balanced ratio of genders, lower cost, more timely data collection, reliable data, and anonymity of participants. Some of the disadvantages are: unrepresentative samples, lower response rates, financial motivation issues, limited access to certain portals, limited length of study, non-behavioral data, and lack of follow-up data. In addition, the authors share their personal experiences of using these portals and summarize previous literature researching online populations.
\end{abstract}

Keywords: online research, participants, research methods

\section{Introduction}

Since the earliest days of aviation, research has been used to support the design and analysis of technological designs and enhance pilot behavior. Traditional models of the pilot in the cockpit or within a flight simulator have given way to new methods of investigation and data collection. With the advent of new technology has also come new methods of obtaining data within the research community, such as through direct targeting of certain populations in chatrooms or message boards, online crowdsourcing in which participants back a project to be completed and then contribute to the data collection, and internet-based survey instruments. These methods allow aviation researchers to collect broader and novel data that may have been unavailable to them in previous years.

This article more specifically focuses on the method of internet-based survey administration. Two more common platforms for online survey administration are SurveyMonkey ${ }^{\circledR}$ and Amazon's Mechanical Turk ${ }^{\circledR}$ (MTurk). These two platforms allow the researcher to develop and administer surveys to target populations indexed by specific criteria in the survey respondents. This allows the researcher to obtain large volumes of survey data quickly and cheaply compared to more traditional survey methods. However, those methods also come with some potential drawbacks. This article discusses some of the advantages and disadvantages of internet-based surveys for data collection in the aviation realm.

All correspondence concerning this article should be directed to Stephen Rice at scrice@ outlook.com. 
Problem Statement: Gaining Access to Participants and the Use of Online Populations

A challenge somewhat unique to conducting research in aviation can be getting access to participants. In some disciplines, access can be easily obtained, but within the constructs of aviation research, a major challenge to the research process can be getting access to populations from which to sample. Many aviation organizations have heavy restrictions or are even unwilling to send out instruments on behalf of researchers to protect their members from unwanted solicitations. Companies, such as airlines, may have concerns over the use of their data, trade secrets or proprietary information. Labor organizations may also want to screen or limit access to their populations. Some or all of these obstacles could be a major challenge to researchers. However, within the last decade or so, there has been the creation of online populations that provide access to individuals who are interested and willing to participate in research studies. These online populations provide a unique opportunity to collect data related to aviation issues. The purpose of this paper is to provide a summary on the advantages and disadvantages of using these populations for researchers to help them determine if these populations are a viable source of data collection for future studies and the research questions seek to identify what are the advantages and disadvantages of using online populations for research in aviation. Throughout the paper, comparisons between the use of online populations and studies conducted in face-to-face settings will be discussed. The goal of this is to provide a benchmark for comparison: a traditional face-to-face setting (the laboratory) and the new setting (online populations).

\section{Methodology: Advantages of Internet-Based Research Methods in Aviation-Related Research}

With the advent of internet-based surveys, a wide array of positive effects are offered for the aviation researcher. These benefits include access to new and broader populations with a wider base of characteristics, the ability to collect larger volumes of quality data than previous survey methods which enhances confidence in research study conclusions, savings in time and cost, and the ability of participants to take part anonymously which means more participants may be willing to participate. Online populations are those groups of individuals who are willing to complete various tasks online and are associated with structured organizations such as SurveyMonkey ${ }^{\circledR}$ or Amazon's MTurk.

\section{Easy Access to (New) Populations}

An immediate benefit of internet-based research methods in aviation is the access to new populations that were previously unavailable before this approach. The majority of aviation research is limited to an institution's population of students or faculty, the majority of whom are American (Haddock \& Beckman, 2015; Littlepage, Hein, Moffett, Craig, \& Georgiou, 2016; Wang, Anne, \& Ropp, 2016; Wilson, Bjerke, \& Martin, 2015). The published data in this field are often limited because they are Western, educated, industrialized, rich, and democratic (WEIRD), and are often not representative of other countries or cultures (Henrich, Heine, \& Norenzayan, 2010). Having WEIRD data is not a problem if the main objective includes generalizing the results to other institutes or similar populations. However, researchers may want to improve their study's external validity, and gathering data from a population outside an institute's or university's resources quickly becomes challenging. Despite these challenges, new avenues for access to certain targeted populations become available through internet-based survey methods.

For example, when the authors began researching consumer perceptions of autopilots in commercial aviation (Rice et al., 2014), it quickly became apparent that finding an accessible population would be difficult. The researchers considered passing out questionnaires at local airports, but the airport authority denied their requests. Furthermore, most passengers do not want to be approached by a stranger in an airport and asked to fill out a questionnaire. The researchers also contemplated sending interviewers to public places to gather data, but they did not know if the data would generalize to the intended target group of people at airports. Fortunately, the use of internet-based research method enabled the researchers to gather data from 1,000 qualified participants in three hours, effectively saving the researchers a significant amount of time and money.

In another example that illustrates how new populations can be reached through internet-based survey methods, the researchers were also interested in comparing different cultures' perceptions of autopilots in commercial aircraft, in particular, people from India; however, they had little access to India's population. Two of their graduate students were from India so, with sufficient funding, the graduate students could have returned to India to collect data; however, they would only have access to a small percentage of people in Bombay. There was no possible way for the students to gather data from Indians living in other cities, towns, or villages. In contrast, there are millions of Indians with access to the internet who are willing to fill out opinion surveys during their leisure time. Thus, with access to online populations, the researchers gathered data from around 200 participants in both the United States and India. In follow-up studies (Rice \& Winter, 2015; Winter \& Rice, 2015; Winter et al., 2015), researchers collected data from over 5,000 Indians, the majority of whom all resided in different provinces. Also, other researchers have collected data in countries throughout most of Europe, South America, Africa, Asia, and Greenland. Prior to this method, it was highly infeasible to tap into those markets without having friends or 
colleagues at local universities. Furthermore, researchers would likely only receive access to the students at those universities. Online populations allow for a much broader base of participants to be reached compared to traditional methods.

\section{Greater Generalization}

When conducting scientific research, it is critical that one can generalize the findings to the larger population from which one is sampling. When generalizing, researchers must be cognizant of the difference between the target population and the accessible population. The target population includes the group of people researchers want to sample from, and the accessible population is the group of people researchers have the ability to access, and do, a sample from. For example, if one is trying to determine the perceptions of the American public to privacy issues during unmanned aerial vehicle (UAV) flight (Winter, Rice, Tamilselvan, \& Tokarski, 2016), the target population includes Americans. However, since it is infeasible to gather a random sample of all Americans, researchers limit themselves to an accessible population from which they can collect a reasonable sample size.

In the past, most aviation-related research used samples taken from local universities, institutes, flight schools, etc. Thus, the accessible populations were limited in scope to one or two particular institutions, and only those participants had a chance to be a part of the study. This weakens a study's external validity, and critics argue that if one samples from undergraduate students at a university then one can only generalize to undergraduate students at that university. The results would not even generalize to undergraduates at other universities because researchers had no evidence that other universities contained identical populations. Using internet-based data collection does not completely alleviate this problem; however, when done correctly, it creates stronger external validity, assuming the sample data were collected from a larger accessible population.

\section{Age and Gender of Participants}

In addition to easily expanding the accessible population that one would like to sample from, it is also important for researchers to sample from a large age range (Field, 2013). However, it is acknowledged that this is also delimited by the purpose of individual studies. In the previous example regarding UAV privacy perceptions, a researcher would be interested in knowing what all age groups feel about UAV privacy issues. If the only sample collected includes college-aged students, then the age range may be limited and distorted. The data are limited because most college students are 18-30 years old; and it is distorted because the few students who are over 30 cause an unbalanced weighting in the high range, creating a challenge in making accurate statistical conclusions regarding age differences. It would be more ideal to collect data from a pool of participants who have a broader, and normally distributed, age range. While the mean age of college participants tends to fall between 19 and 20 years of age, the average age of participants in online populations tends to fall between 30 and 35 years of age, which is closer to the mean age of pilots and aviation consumers in general, and therefore more representative of the target population.

Furthermore, gender is also an important issue in research (Field, 2013), and particularly in aviation-related research, but, considering only around 6\% of pilots are female, this group presents a challenge for data collection. Many of the universities with flight schools have gender imbalances as well, with far more male students than female students, which can skew sampling results. Moving the research questionnaires online provides a more balanced gender ratio, with many online survey services offering an almost 50-50 ratio of females to males for non-pilot aviation consumers. Gender matters in aviation research, as illustrated in published studies that demonstrate males and females differ across many tasks and paradigms.

Importantly, many online populations allow the researcher to specifically target certain genders, countries, and age groups. If researchers need female Germans who are $18+$ years old, then it is relatively simple to open their study to only those participants who qualify.

\section{Larger Sample Sizes}

Bigger samples provide better generalization to research, reduce variance, and increase power in statistical analyses while providing more flexibility when it comes to violation of assumptions in parametric tests. Importantly, larger samples provide more accurate information about effect sizes. Due to these factors, it is preferable to conduct research with large sample sizes.

When conducting aviation-related research, it is often difficult to find enough participants to fulfill power analyses requirements. Students often complain that their theses and dissertations are prolonged because they cannot find enough participants for their studies. This is particularly difficult when the study requires specific expertise in participants (e.g. pilots), but it is also problematic when tapping into the general population of consumers or passengers. Part of this problem lies in the number of participants who are available for the study.

The ability to tap into an online source of participants provides an outlet that solves the participant problem, particularly if the researchers are not concerned about specific populations of experts. SurveyMonkey ${ }^{\circledR}$ and Amazon's MTurk are two of the largest sources of online participants, and both boast populations in the millions of people. With access to these large populations, researchers can collect data from hundreds or even thousands of participants per hour. Several recent doctoral students (Cremer, 2015; Ozyurek, 2016; Rosser, 2016) have taken advantage of this 
participant pool, and they reveal sample sizes close to 1,000 participants in their studies.

\section{Time}

Timely research is critical, particularly in aviation, because the information derived from sound research needs to be disseminated as quickly as possible to government and industry partners. Many aviation researchers and those in other related fields complain that the research process takes too long from idea germination to publication (Mason \& Suri, 2012). Unfortunately, the findings are sometimes published long after the technology is obsolete. One factor causing a delay in the research process is due to the timeconsuming nature of data collection. When using traditional data collection procedures, researchers must bring participants to the laboratory to collect data from them. Then, even with the help of graduate and undergraduate assistants, data collection can take several weeks or months, depending on the number of participants. In extreme cases, studies designed to run one participant at a time have taken more than a year to complete data collection.

Internet-based research allows a researcher to collect data from hundreds, and even thousands, of participants in a relatively short amount of time. Importantly, the amount of time is not necessarily dependent on the number of participants. Consider, for example, a recent experience of the authors. When having to complete major revisions on a manuscript under consideration for publication, it was possible to run thousands of participants in parallel when using MTurk (or other online participant pools). In using a traditional laboratory setting for this study, a 10-minute study would take 167 hours to run 1,000 participants. This same study can be done online in 10 minutes (although it usually takes about an hour to collect that much data since not everyone starts at the same time).

\section{Cost}

In addition to collecting thousands of participants per hour using internet-based research, it is often more economical to do so. Studies conducted in the laboratory usually require paying participants for at least one hour of time, which is about $\$ 8-\$ 10$. In fact, many university institutional review boards require the one-hour minimum; therefore, even if one is conducting a 10-minute study, one might still be required to pay $\$ 8-\$ 10$ per participant for their time, as anything lower will not attract enough potential participants. Internet-based research allows a researcher to pay participants only for the time they are working on the study. It is quite common to pay participants $\$ 1$ or less for a 10-minute study on MTurk. SurveyMonkey charges more for this service, but it is still low-priced compared to laboratory collection (Buhrmester, Kwang, \& Gosling, 2011; Paolacci, Chandler, \& Ipeirotis, 2010). For example, one dissertation student recently collected data from over 1,000 participants for $\$ 450$ (Rosser, 2016).

In addition, using online populations eliminates the need to retrieve participants from university subject pools. These subject pools have become controversial in the past decade because it is widely believed that forcing participants to take part in research studies as a requirement of their course grade is a form of coercion (Elliot, Rice, Trafimov, Madson, \& Hipshur, 2010). Some schools have mitigated this problem by allowing students to complete other tasks instead. However, those tasks are usually less interesting and are often intended to be difficult so that students will want to participate in the research studies instead.

\section{The Quality of Data}

The quality of data from university subject pools can be questionable, as well, particularly at the end of a semester when students are struggling to finish their assignments and prepare for finals. Students perceive themselves as having more important priorities than participating in a researcher's laboratory and they may not put forth all of the effort and attention the study deserves. Paying online participants can eliminate the need to access university subject pools (when plausible for the type of study), and provides access to participants who want to be involved in the research study.

The quality of data is one of the most important concerns regarding non-laboratory research or research not conducted in a face-to-face setting, and one that must be sufficiently addressed before using alternative methods of data collection. While not every online population for data collection has been tested for data reliability, there have been several notable studies conducted on MTurk, and other sites, and their results suggest that the data collected on these participant portals are as reliable as data that could be collected in a traditional laboratory setting (Berinsky, Huber, \& Lenz, 2012; Buhrmester et al., 2011; Germine et al., 2012; Mason \& Suri, 2012).

There is an additional benefit to internet-based data collection. Many sites allow the researcher to rate participant performance subjectively. If participants do not fulfill the tasks, the sites allow the researcher to reject their work with non-payment or issue a poor rating. In addition, MTurk allows researchers to filter potential participants based on a set of pre-determined criteria, and one of these criteria is the performance rating they have received from previous researchers. Over time, these approval/rejection evaluations add up to an effective rating system whereby researchers have access to the percentage of prior tasks approved for each participant. For example, if one wants to ensure high-quality participants, then one can choose only to accept participants who achieved a $95 \%$ or higher approval rating. This ensures that the people one is paying to collect data from have been approved $95 \%$ of the time in previous tasks. Figure 1 shows an example of a few options available to researchers in the selection of their participants. 


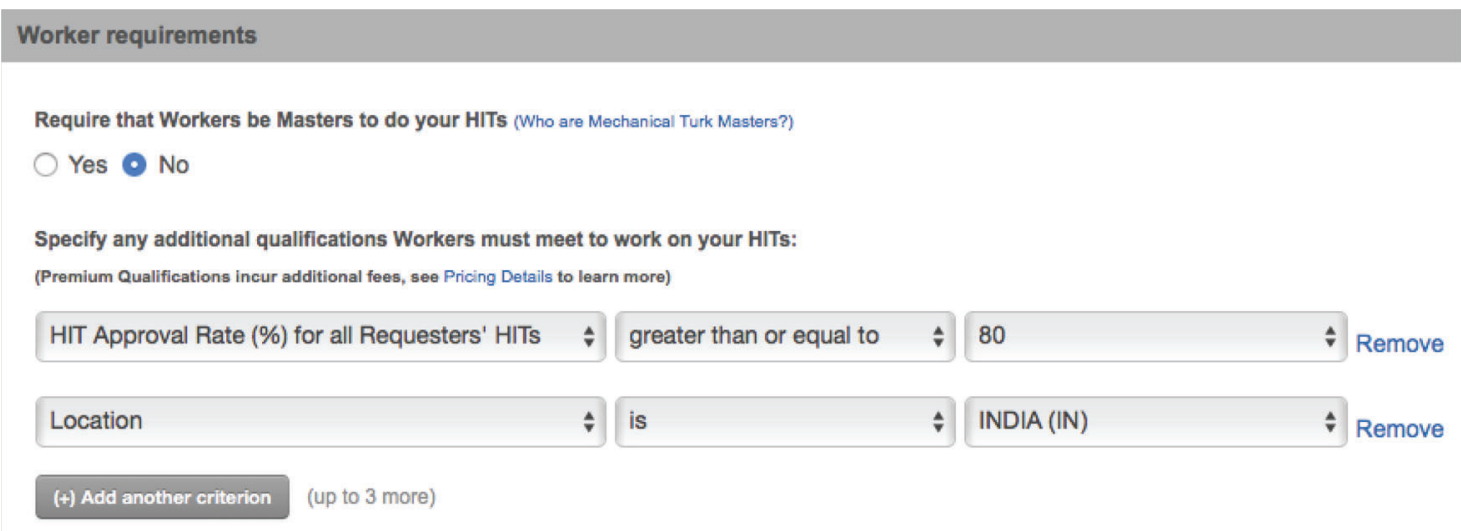

Figure 1. Screenshot of the worker (participant) requirements selection criteria from MTurk. Researchers can select worker approval ratings (in this example, 80 or better), location, and other parameters as necessary.

\section{Anonymity}

When conducting certain studies, it is critical to ensure that participants retain their anonymity throughout the research study as many participants will not provide honest data if they feel their responses can be tied back to them personally. Some online participant portals do not allow researchers access to the identity of the participants, thus ensuring their anonymity and protecting the participants. Depending on the type of study being conducted, if participants remain anonymous then this can help meet the requirements as established by an institutional review board. If more information is necessary from participants, researchers can design a study where tracking methods are established.

\section{Disadvantages of Internet-Based Research Methods in Aviation-Related Research}

While there are plenty of positive benefits in using internet-based research methods for data collection, there are also some drawbacks that suggest these methods should be used with caution despite the benefits. Non-representative samples, low response rates, financial motivation, participant fraud, limitations in types of data collected, and practical survey administration issues all impact the use of online survey data collection.

\section{Non-Representative Samples}

A disadvantage of internet-based research includes the inability to collect a truly random sample of the U.S. population. A random sample must allow for each of the potential participants to have an equal chance at participating in reducing bias in selection in participants; however, there are at least four ways that internet-based research renders random sampling impossible. To begin with, only people who sign up for the websites that promote these online surveys will have a chance at participating (Mason $\&$ Suri, 2012). All other members of the target population have no chance of participating because they are not viewing the websites that provide access to these surveys. Therefore, important viewpoints or key data may end up missing in the final data set because those critical values were not represented.

Second, only those participants who happen to be online at the exact time the study is offered can participate. For example, a person might be a member of MTurk, but does not $\log$ in until after work hours; this person will miss all the studies offered in mornings or lunch times (Mason \& Suri, 2012). This obstacle can be particularly difficult when trying to collect data from other countries because a researcher needs to consider the schedules of participants living in foreign time zones. Some researchers have solved this problem by posting their studies online and leaving them overnight, so those living in different time zones have ample time to complete their survey. Another potential solution to this problem entails offering the study during different times of day; therefore, everyone has a chance to participate. Unfortunately, this solution does not alleviate the third problem.

The third problem involves an inherent bias in survey respondents. When a study is posted on MTurk, only the participants who respond the fastest will complete the study. For example, imagine posting a new study on MTurk looking for 200 participants; at any given moment, there may be over one million people on MTurk who might be interested. However, only the first 200 will be allowed access to the study because once the study has met its quota, it will close. This could be troublesome when trying to find participants from Asia, as the researcher now has to stay up all night posting the study throughout different times during the Asian day, or else risk getting all 200 participants from a 10-minute time window.

Lastly, online populations are most likely not part of the aviation industry (Buhrmester et al., 2011; Paolacci et al., 2010). While it is easy to find aviation consumers online, most of the participant portals do not offer much access to aviation experts (e.g. pilots, crew, maintainers). If one needs aviation experts to fill out questionnaires, then online population pools would not be a wise choice. 
Instead, these researchers would do better by sending an email to potential participants and include a link to the survey via that route.

Clearly, gathering a perfectly random sample is not possible when using internet-based research unless the researcher sets radical limitations for the target population. Some researchers choose to follow this route, but others simply admit that they are utilizing convenience sampling that includes a trade-off of ease of participant access for potential response bias (Berinsky et al., 2012). Fortunately, convenience sampling highlights the large diversity that can be achieved compared to traditional settings in which researchers sample only from a certain group of participants.

\section{Response Rates}

Face-to-face studies typically enjoy high response rates for participants. If a participant is willing to show up for a study, it is likely they will stay for the entire study, and the researcher will have the ability to collect a full set of data. Obviously, there are a few who quit halfway through, but the vast majority of participants continue unless the task is noxious. There are multiple reasons for the retention rate, but one factor is probably that people are less willing to walk out on a study where they are face-to-face with the experimenter. Walking out means a confrontation, albeit a non-threatening one.

Unfortunately, internet-based research does not enjoy the high response rate that laboratory studies maintain. Online participants frequently start a study and then quit after the first few questions. They often review a study to see if it is interesting, and then if it does not seem appealing, they will often quit after the instructions (Buhrmester et al., 2011). They are also much more likely to quit in the middle of the study, particularly if the experimenter has not created page numbers or progress completion reports along the way. In the researchers' experience, about $10 \%$ of participants quit after reading the instructions. Another 10-15\% quit halfway through the study although this percentage varies dramatically depending on the length of the study. A short one- to five-minute study might retain $95 \%$ of participants; whereas a fifteen-minute or longer study might retain only $50 \%$ of participants. Much of the retention rate depends on factors like interest in the study, amount of pay, and length of the study (Buhrmester et al., 2011). Thus, it is important for researchers to understand these factors and plan accordingly.

\section{Financial Motivation and Potential Fraud}

There are many different motivations for participants to take part in a research study. One of the most common is the subject pool that many universities use to collect research data (Henry, 2008; Sears, 1986). In subject pools, students are required to participate in experiments as part of their course grade. Another motive includes the two different types of compensation provided to participants volunteering their time for a study. Many researchers will pay their participants for their time and effort; however, some researchers ask for participants to volunteer their time with no compensation freely. In the latter situation, a participant may agree if they believe they are helping with an important study. In fact, many pilots and other aviation professionals are frequently willing to fill out surveys for free.

Unfortunately, financial compensation may have some negative side effects depending on participants' expectations. Participants are often aware that they are receiving compensation. Therefore they want to maximize their pay/ time ratio. Thus, many of them will rush through a study just to collect their payment. Some will even pretend to complete the study, but not answer as accurately as possible (e.g. answer the same response throughout the survey), and then expect payment. While fraud is not rampant on most internet-based data collection sites (Berinsky et al., 2012), it is still present in some cases. Researchers should be wary of this and provide checks and balances in their study to make sure the participants are doing their best, such as having careful survey construction to enable reliability assessments of participant respondents. Some sites offer rating systems, which help researchers find the most highly rated participants for their studies.

\section{Access Limited to Certain Portals}

A big dream for researchers includes finding a resource where they can access an unlimited number of participants for their studies. Although internet-based data collection sites provide millions of potential participants, there are a limited number of sites like this, and it is questionable how many of the participants on one site are also members on other sites. In fact, given the demographics of online populations, it is very likely that many of them are members of multiple sites. This may not be a problem in the short term, but eventually demand will catch up with supply, and the cost of conducting online research will increase (Peer, Brandimarte, Samat, \& Acquisti, 2017). In fact, the cost has already risen by $1000 \%$ or more on MTurk. As recently as 2014, it was possible to pay participants 1 US cent for a 10-question study (Buhrmester et al., 2011), and still receive rapid response rates. Now, the bare minimum appears to be 10 cents but is usually more in the $20-50$ cent range. Granted, this remains a very low cost in comparison to other data collection sources, but it will be interesting to see how much it rises over the next few years.

\section{Time Participants are Willing to Give}

When bringing subjects into a face-to-face setting to conduct aviation research, it is often possible to earn 1-4 solid hours out of a paid participant; however, online 
research is much more limited in this aspect. Many researchers have reported that they have trouble getting online participants to spend more than 20-30 minutes on a survey before they either quit or start responding randomly. In the same way, this also limits a researcher's ability to collect accurate data from a time-consuming survey (Crump, McDonnell, \& Gureckis, 2013).

\section{Usually Measures Attitudes and Perceptions, Not Behaviors}

Online research is also limited by the types of participants that can be recruited and the types of questions they can be asked. As stated earlier, the types of persons comprising these online populations are usually not experts in a particular field, such as pilots or aviation mechanics. Even if the researchers attempted to scope the study and only request individuals with those types of qualifications, it is impossible to verify, with any reliability, that participants met those qualifications (Crump et al., 2013). Therefore, research studies using these populations are limited in that they, for the most part, can only gather data concerning attitudes and perceptions; although sometimes it is possible to link to a site where one might collect behavioral data.

\section{Instrument Administration}

The ability for a researcher to verbally instruct participants and answer questions during the research process greatly enhances the participants' understanding of the task they need to complete. However, this communication becomes much more difficult online, where the researchers and participants never meet (Crump et al., 2013). Thus, researchers need to be very clear and detailed with their instructions, while also being cognizant of the fact that some participants may not read the instructions at all or only read the first few lines. For more detailed studies, some researchers have created a few practice/screening questions that participants must complete to progress to the actual assessment (Crump et al., 2013). If participants do not successfully complete the practice session, they are dismissed from the study. Additionally, formal consent forms in which a participant can explicitly provide consent to participate in the research are typically absent in research of this nature because the researcher and participant are not collocated and finding a witness to observe the participant is typically not cost effective. Consent is typically provided through participation in the study, but there is frequently no method for the participant to verbally or electronically explicitly provide their consent for the study participation. Conversely, when the participant is finished, it is often difficult to provide debriefing of the nature of the study to the participant as the only method to outline the true nature of the study to the participant is through a written statement at the end of the instrument that the participant may not read and therefore the researcher has no knowledge if the respondent truly understands what they have participated in. Consent and debriefing are critical elements to safeguard both the participant and researcher and must be assumed in the administration to online populations.

\section{Lack of Follow-Up Ability}

Many online populations do not provide the researcher with knowledge of the participants' identity, making it impossible to invite them back for another study (Crump et al., 2013). If a researcher plans to conduct a longitudinal study, or a test-retest type study, they may want to seek alternative participant pools.

Additionally, when using online populations, researchers cannot complete behavioral studies or studies that would require repeated measures, such as a training type study. Since participants are not physically in a face-to-face setting, they cannot be placed in simulators nor can other equipment such as eye trackers be used. Therefore, researchers must create hypothetical scenarios to evaluate participant responses. Also, due to the vast nature of these online populations, it would be challenging for researchers to complete a study, and then have participants return to complete another assessment at a later time. Furthermore, it would be difficult for the researchers to verify that the participant completing the follow-up assessment was in fact the same individual that completed it the first time. These factors suggest that while there are a great many benefits of the use of online populations there also must be caution taken in administration and interpretation of these results.

\section{Analysis/Discussion}

Internet-based research offers both advantages and disadvantages to aviation researchers. It provides access to diverse participants who might not otherwise be accessible to researchers. This may help with the generalizability of studies, which may be limited if only using traditional university students. Using online portals also allows for data to be collected quickly, often within a few hours as opposed to days, weeks, or months. In addition, it is economically more advantageous because participants are paid $20-50$ cents per hour rather than $\$ 8-\$ 10$ per hour in a laboratory.

However, there are also disadvantages due to random sampling validity, response rates, and limitations on gathering behavioral data. Researchers cannot obtain a truly random sample of participants as they are limited to those that have subscripted to the service, are active, and are available at the time the researchers post the instrument. Response rates can vary depending on the length of the survey, and researchers have to remain aware of and take precautions to prevent fraudulent data. Online populations also limit the type of studies that can be completed as it would be very 
difficult to obtain behavioral data or conduct studies that would require repeated measures.

These online populations provide a new alternative for researchers in the aviation field. As with all populations, online populations have advantages and disadvantages, and these tradeoffs must be assessed by each researcher to determine if their use is appropriate for the area under investigation. The purpose of this paper was to provide a comprehensive analysis of the advantages and disadvantages to assist researchers in this assessment.

\section{Conclusions}

In summary, online populations provide an alternate perspective and access to another population, which has its own delimitations and limitations. Like all studies, it is important to understand the advantages and disadvantages of using various populations for research. Clearly, there are some studies that would not be feasible or appropriate to complete with online populations; but there are a number of studies across several disciplines that could benefit from these tools. This paper has provided information on both advantages and disadvantages so researchers can make informed decisions when conducting their aviation research studies.

\section{References}

Berinsky, A. J., Huber, G. A., \& Lenz, G. S. (2012). Evaluating online labor markets for experimental research: Amazon.com's Mechanical Turk. Political Analysis, 20, 351-368.

Buhrmester, M., Kwang, T., \& Gosling, S. D. (2011). Amazon's Mechanical Turk: A new source of inexpensive, yet high-quality, data? Perspectives on Psychological Science, 6(1), 3-5.

Cremer, I. (2015). Creating and validating a perception of airport sustainability (PASS) scale (Unpublished doctoral dissertation). Florida Institute of Technology, Melbourne, FL.

Crump, M. J., McDonnell, J. V., \& Gureckis, T. M. (2013). Evaluating Amazon's Mechanical Turk as a tool for experimental behavioral research. PLOS ONE, 8(3), e57410. https://doi.org/10.1371/journal. pone.0057410

Elliot, L., Rice, S., Trafimow, D., Madson, L., \& Hipshur, M. (2010). Research participation versus classroom lecture: A comparison of student learning. Teaching of Psychology, 37(2), 1-3.

Field, A. (2013). Discovering statistics using IBM SPSS statistics. London: Sage.

Germine, L., Nakayama, K., Duchaine, B. C., Chabris, C. F., Chatterjee, G., \& Wilmer, J. B. (2012). Is the web as good as the lab? Comparable performance from web and lab in cognitive/perceptual experiments. Psychonomic Bulletin \& Review, 19(5), 847-857. https://doi.org/ 10.3758/s13423-012-0296-9

Haddock, K. N., \& Beckman, W. S. (2015). The effect of electronic flight bag use on pilot performance during an instrument approach Collegiate Aviation Review, 33(1), 1-13.

Henrich, J., Heine, S. J., \& Norenzayan, A. (2010). The weirdest people in the world? Behavioral and Brain Sciences, 33, 61-135.

Henry, P. J. (2008). College sophomores in the laboratory redux: Influences of a narrow data base on social psychology's view of the nature of prejudice. Psychological Inquiry, 19, 49-71. https://doi.org/ 10.1080/10478400802049936
Littlepage, G. E., Hein, M. B., Moffett III, R. G., Craig, P. A., \& Georgiou, A. M. (2016). Team training for dynamic cross-functional teams in aviation: Behavioral, cognitive, and performance outcomes. Human Factors, 58(8), 1275-1288.

Mason, W., \& Suri, S. (2012). Conducting behavioral research on Amazon's Mechanical Turk. Behavioral Research, 44, 1-23.

Ozyurek, A. S. (2016). An examination of how ratings of airlines are affected by different types of information: A mediation analysis (Unpublished doctoral dissertation). Florida Institute of Technology, Melbourne, FL.

Paolacci, G., Chandler, J., \& Ipeirotis, P. G. (2010) Running experiments on Amazon Mechanical Turk. Judgment and Decision Making, 5(5), 411-419.

Peer, E., Brandimarte, L., Samat, S., \& Acquisti, A. (2017). Beyond the Turk: Alternative platforms for crowdsourcing behavioral research. Journal of Experimental Social Psychology, 70, 153-163.

Rice, S., Kraemer, K., Winter, S. R., Mehta, R., Dunbar, V., Rosser, T., \& Moore, J. (2014). Passengers from India and the United States have differential opinions about autonomous auto-pilots for commercial flights. International Journal of Aviation, Aeronautics, and Aerospace, 1(1), 1-12.

Rice, S., \& Winter, S. R. (2015). Which passenger emotions mediate the relationship between type of pilot configuration and willingness to fly in commercial aviation. Aviation Psychology and Applied Human Factors, 5(2), 83-92.

Rosser, T. G. (2016) Which emotions mediate the relationship between type of pilot training and willingness to fly in commercial aircraft (Unpublished doctoral dissertation). Florida Institute of Technology, Melbourne, FL.

Sears, D. O. (1986). College sophomores in the laboratory: Influences of a narrow data base on social psychology's view of human nature. Journal of Personality and Social Psychology, 51(3), 515-530.

Wang, Y., Anne, A., \& Ropp, T. (2016). Applying the technology acceptance model to understand aviation students' perceptions toward augmented reality maintenance training instruction. International Journal of Aviation, Aeronautics, and Aerospace, 3(4), 1-13.

Wilson, N., Bjerke, E., \& Martin, L. (2015). Aviation living learning community: Impacts on student success. Collegiate Aviation Review, 33(1), 29-43.

Winter, S. R., \& Rice, S. (2015). Pilots who are perceived as unsociable are perceived as more likely to have a mental illness. Aviation Psychology and Applied Human Factors, 5(1), 36-44.

Winter, S. R., Rice, S., Mehta, R., Cremer, I., Reid, K. M., Rosser, T. G., \& Moore, J. (2015). Indian and American consumer perceptions of cockpit configuration policy. Journal of Air Transport Management, 42, 226-231.

Winter, S. R., Rice, S., Tamilselvan, G., \& Tokarski, R. (2016). Missionbased citizen views on UAV usage and privacy: An affective perspective. Journal of Unmanned Vehicle Systems, 4, 1-11.

Dr. Stephen Rice is an associate professor at Embry-Riddle Aeronautical University, Daytona Beach. He received his $\mathrm{PhD}$ in human factors from the University of Illinois at Urbana-Champaign.

Dr. Scott R. Winter is an assistant professor at Embry-Riddle Aeronautical University. He received his $\mathrm{PhD}$ in aviation technology from Purdue University.

Dr. Shawn Doherty is an associate professor at Embry-Riddle Aeronautical University, Daytona Beach. He received his $\mathrm{PhD}$ in human factors from the University of Illinois at Urbana-Champaign.

Ms. Mattie Milner is a master's student in human factors at Embry-Riddle Aeronautical University, Daytona Beach. She received her bachelor's degree from Centenary College of Louisiana. 